\section{Detección de brucelosis humana en pacientes de Sinaloa, México, en 2006}

Señor editor: La brucelosis tiene grandes repercusiones en la salud de los seres humanos y de los animales de crianza en todo el mundo. Representa un importante problema sanitario y económico. ${ }^{1,2}$ En México, la brucelosis humana es una zoonosis muy frecuente. Tan sólo en 2003 se registraron 2945 casos, de los cuales $19.45 \%$ ( $n=573)$, se presentaron en el estado de Sinaloa, cifras que colocaron a dicha entidad con el mayor número de casos humanos en todo el país, ${ }^{3}$ constituyéndose así en un problema de salud pública. Sin embargo, no existen estudios recientes que indiquen el grado de endemia existente ni el perfil epidemiológico de la enfermedad en México y en particular en Sinaloa. En este contexto, se buscó estimar la seroprevalencia de la brucelosis humana en muestras de pacientes que acudieron al Centro Estatal de Transfusión Sanguínea de Sinaloa y a los laboratorios de la Facultad de Ciencias Químico Biológicas de la Universidad Autónoma de Sinaloa (UAS).

Efectuamos un análisis epidemiológico para identificar factores de riesgo asociados con un definitivo impacto en la morbilidad, derivada de la brucelosis humana. Las muestras séricas fueron obtenidas de individuos que acudieron a los laboratorios de la Facultad de Química de la UAS, para la obtención de un certificado médico, o al Banco de Sangre del Centro Estatal de Transfusión Sanguínea de Mazatlán, Sinaloa, como donadores potenciales y que dieron su consentimiento por escrito de acuerdo con los lineamientos del Comité de Ética de ambas instituciones. Las muestras séricas de los individuos participantes, de ambos géneros, con un rango de edad de 15 a 65 años, fueron colectadas durante el periodo comprendido de enero a diciembre de 2006. A todas las muestras séricas se les realizaron las siguientes pruebas serológicas: Rosa de Bengala (RBT, BioRad México), Microaglutinación (MAT,
Interbiol, México), y 2-Mercaptoetanol anti-Brucella (2-MET, Micsa México), usando Brucella abortus como antígeno de acuerdo con los procedimientos descritos por los fabricantes y siguiendo los criterios de la Norma Oficial Mexicana correspondiente. ${ }^{4}$ Para la identificación de los factores de riesgo asociados con la brucelosis en Sinaloa se realizó una encuesta estructurada sobre hábitos higiénico-alimentarios y ocupacionales de cada individuo incluido en este estudio. La información se almacenó en una base de datos diseñada para tal fin, utilizando para ello el programa de cómputo Excel 2007. Una vez verificada la calidad de la información se procedió a organizar y analizar la información utilizando para ello el software Stata Intercooled versión 8 .

Se colectaron 1025 muestras séricas pareadas con su respectiva encuesta. El $59.02 \%(n=605)$ de las muestras correspondió al género masculino. La edad promedio de los individuos que reportaron su edad fue de 22.60 años $(D E=8.26)$. Con respecto al nivel de escolaridad reportado por $97.17 \%$ ( $n=996)$ de los participantes, $43.67 \%$ ( $n=435)$ indicó haber cursado la preparatoria, seguido por secundaria con $34.84 \%$ ( $n=347)$; en tercer lugar se ubicó el nivel profesional con $13.25 \%$ ( $n=132$ ). Lo anterior sugiere que la mayoría de los participantes tiene un buen nivel formativo que puede impactar en sus hábitos higiénicos-alimentarios. En cuanto a la ocupación, en primer lugar encontramos a la de estudiante con $68.35 \% \quad(n=689)$, seguido de los empleados con $14.48 \%$ (n=146). De los participantes sólo 0.89\% $(n=9)$ individuos tienen una ocupación relacionada con el manejo de ganado y sus productos, lo cual coincide con la baja frecuencia de individuos positivos a Brucella spp. Con respecto al consumo de leche bronca, 9.48\% $(n=82)$ de los individuos manifestó consumir ese producto. En tanto que $11.17 \%$ ( $n=107)$ respondieron haber padecido fiebre en los 15 días previos al estudio (tal vez relacionado con otro padecimiento). Únicamente ocho de los participantes refirieron tener un familiar al que le hubieran diagnostica- do brucelosis, lo cual representó $0.90 \%$; sin embargo, ninguno de ellos resultó positivo a ninguna de las pruebas inmunodiagnósticas realizadas.

El 0.19\% ( $n=2)$ fueron inmunológicamente diagnosticados como positivos a anticuerpos específicos anti-Brucella spp. Debido a esta baja frecuencia de individuos diagnosticados serológicamente como positivos a brucelosis humana, no fue posible realizar la identificación de los factores de riesgo asociados con la presencia de anticuerpos específicos antiBrucella. Sin embargo, en su encuesta, los pacientes respondieron haber consumido queso fresco, lo cual sugiere que ello pudo ser su medio de contagio puesto que no manifestaron realizar ninguna otra actividad asociada como un factor de riesgo para la brucelosis reportado en otros estudios. Un aspecto de gran relevancia es que dichos quesos frescos los adquirieron en establecimientos comerciales, lo que sugiere una probable deficiencia en la inspección sanitaria a sitios públicos donde se comercia con derivados lácteos potencialmente transmisores de brucelosis. Esto concuerda con lo encontrado por Villamarín-Vázquez y colaboradores, quienes estudiaron 547 sueros de personas, de los cuales todos eran agricultores, y sobre los factores de riesgo destacaron los consumos ocasionales de leche no procedente de central lechera, y de queso fresco. ${ }^{5}$ Asimismo, la seroprevalencia obtenida es más baja que la reportada en la encuesta nacional de la brucelosis en México. ${ }^{6}$

Nuestro estudio aporta información reciente sobre la seroepidemiología de la brucelosis humana en Sinaloa que puede servir de base para futuras investigaciones. Sin embargo, es necesario continuar los estudios de esta enfermedad en otras poblaciones o comunidades del estado. También se desconoce cuál es la especie más frecuente asociada con los casos de brucelosis humana en Sinaloa. Además, podrían implementarse otras metodologías, como ELISA, para un tamizaje mayor, o bien el Coombs anti-Brucella, para identificar los anticuerpos no aglutinantes. 
Es fundamental implementar campañas de difusión sobre la brucelosis humana a fin de informar a la población sobre las medidas profilácticas de esta enfermedad, para disminuir la propagación de la brucelosis humana que afecta cada año a cientos de mexicanos en general y de sinaloenses en particular.

\footnotetext{
López-Moreno Héctor Samuel, QFB, Dr en C,' hsamlo@uas.uasnet.mx Fonseca-Najar Jessica Mirieles, MSP. ' Osuna-Ramírez Ignacio,! Rendón-Maldonado José Guadalupe, MC, Dr en C,' Uribe-Beltrán Magdalena de Jesús, MVZ,' Hernández-Ramírez Carlos Victor. ${ }^{2}$
}

I Facultad de Ciencias Químico Biológicas, Universidad Autónoma de Sinaloa.

${ }^{2}$ Coordinación Estatal de Zoonosis, Secretaría de Salud de Sinaloa.

\section{Referencias}

I. Boletín Epidemiológico 2000;2I(3)septiembre: PAHO.

2. Castro HA, González SR, Prat MI. Brucelosis: una revisión práctica. Acta Bioquím Clín Latinoam 2005;39(2):203-216.
3. Boletín Epidemiológico SSA, 2004.

4. Norma Oficial Mexicana NOM-022-SSA2-

1994

5.Villamarín-Vásquez JL, Chiva-Nevot $F$,

Arnedo-Pena A. Seroprevalencia de la brucelosis en trabajadores agrícolas de las comarcas costeras de Castellón España. Salud Publica Mex 2002;44(2): 137-139.

6. López-Merino A, Migranas-Ortiz R, Perez-

Miravete A, Magos C, Salvatierra IB, Tapia-Coyner $\mathrm{R}$, et al. Seroepidemiología de la Brucelosis en México. Salud Publica Mex 1992;34(2):210-230. 